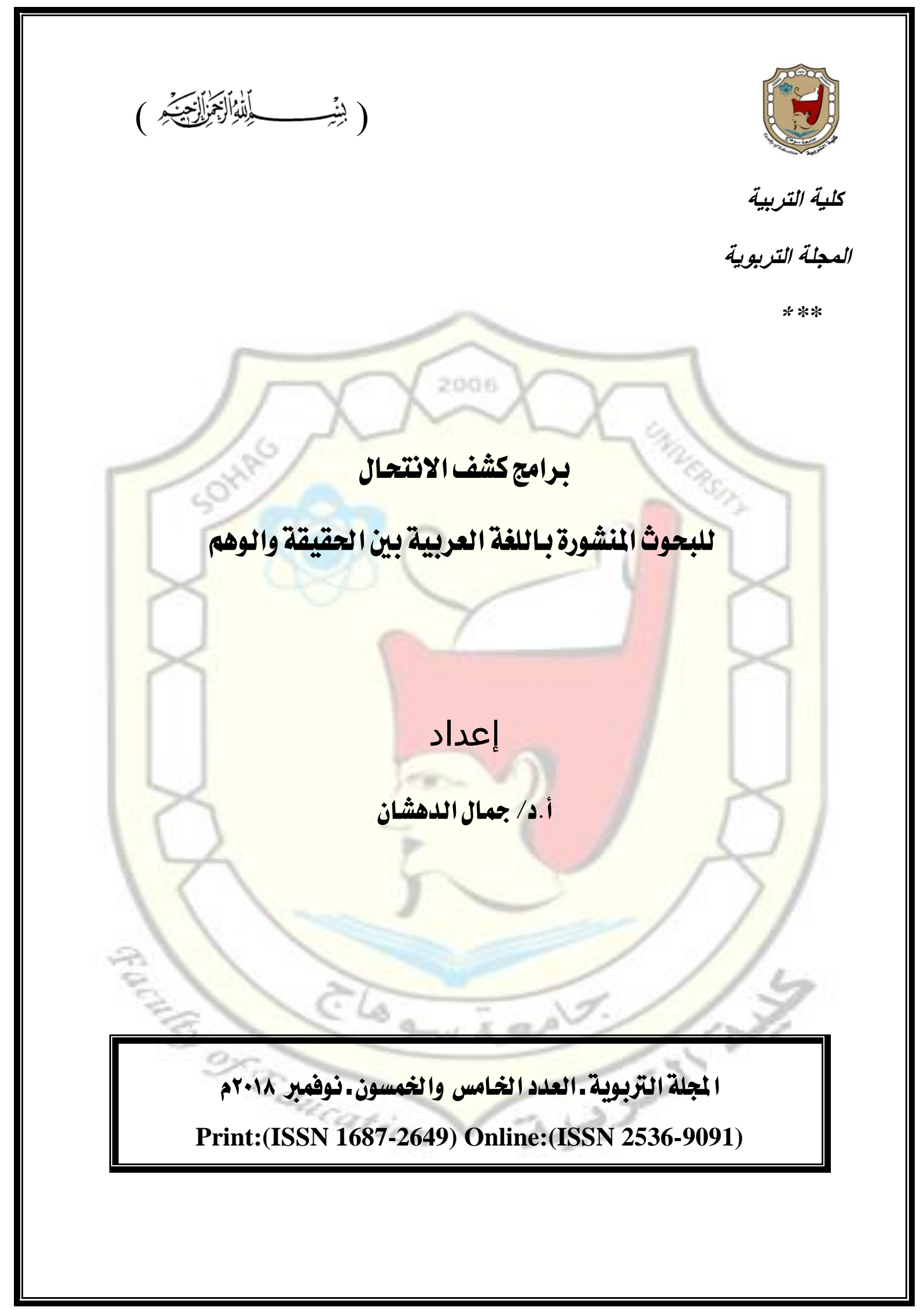


برامج كشف الانتحال للبحوث المنشورة باللغة العربية بين الحقيقة والوهم.

\section{WHAT IS PLAGIARISM?}

Deliberate

Plagiarism

Accidental

Plagiarism

-Rewriting from books or

'not knowing when \&

articles

how to cite

-copying \& pasting

nnot knowing how to

from web pages and

paraphrase or summarize

online sources to create-a

patchwork writing

nnot knowing what

"common knowledge" is

-buying, downloading, or

receycling an old paper

borrowing a paper 


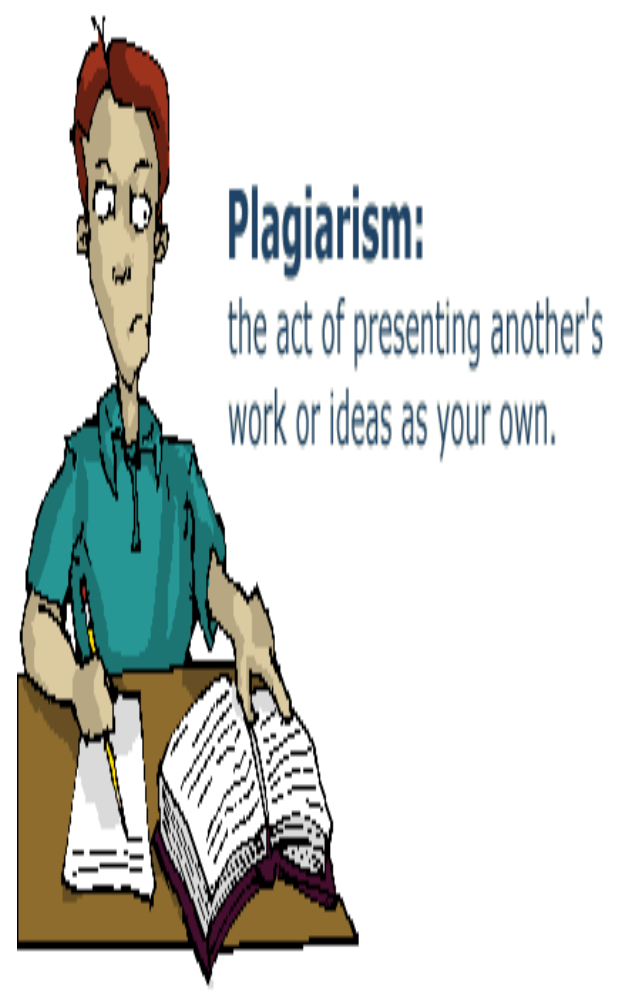

تشهد الساحة العلمية الجامعية ظاهرة جديدة متمثلة فى سعى العديد من الجامعات

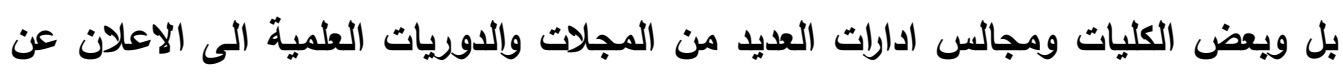

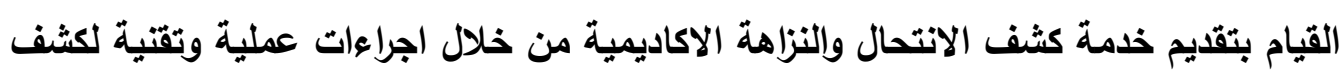
الانتحال والسرقات العلمية مدعية بان لايه اكبر قاعدة بيانات فى العالم ، تطبيقا لمل اشتترطه

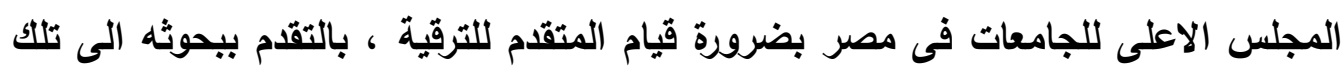
الجهات للحصول على تقرير او شهادة يوضح فيها نسبة الاقتباس من المكتبة الرقمية

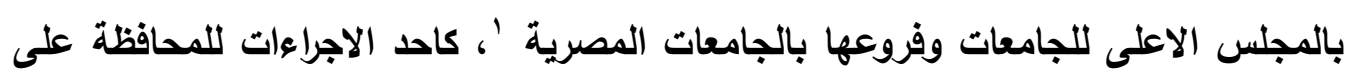
حقوق الملكية الفكرية ومحارية السرقات العلمية والاتتحال العلمي ، وتحديد المصادر التى بالى اقتبس منها ، وكيفية تجنب الوقوع فيه ، حيث نصت قواعد الترقيات بالمجلس الاعلى

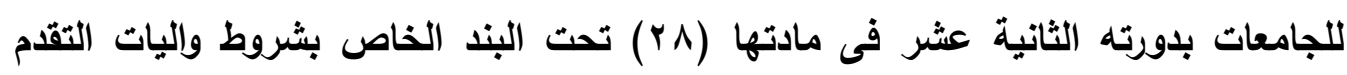

' - انظر ملحق رقم الذى يتضمن دليل اجراءات التقدم لفحص النزاهة الاكا ديمية للابحاث العلمية بالمجلس الاعلى للجامعات 
للجان العلمية الذى يتضمن الاولق والمستثدات المطلوب تقديمها كمتطلبات للتقدم للجان العلمية ضرورة تقديم تقرير معتمد من وحدة المكتبات الرقمية بمركز الخدمات الاكتترونية والمعرفية التابع للمجلس الاعلى للجامعات ، يحدد المادة العلمية المنقولة فى الابحاث

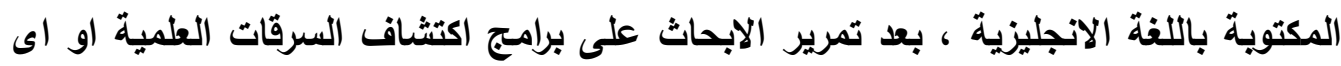

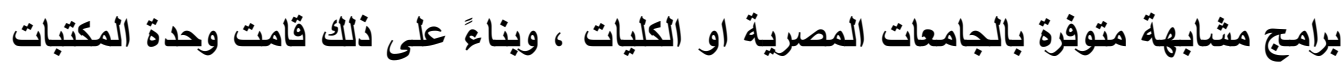
الرقمية بمركز الخدمات الالكترونية بتحديد مجموعة من الضوابط والاجراءات المنظمة للتقدم للفحص تضمنها الملحق رقم (1) كما تضمن نماذج التقدم للحصول على تلك الخدمة ونماذج من التماتج نتائج عمليات الفحص. تللك البرامج التى ظهرت نتيجة ان اعتقاد البعض ان الاعتماد على محركات البحث

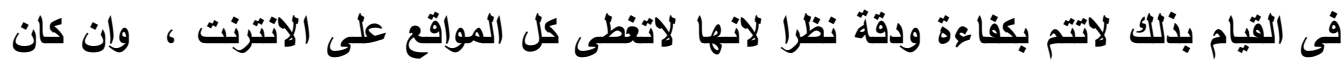

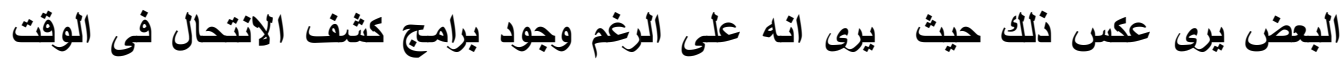

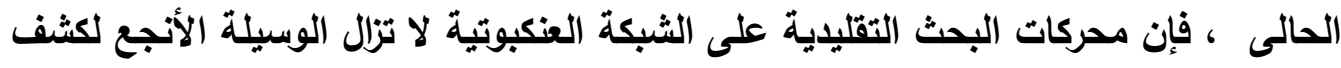
الانتحال السرقات العلمية فى الابحاث المنشورة على الشبكة . وقد فتحت برامجُ الكثف عن الانتحال البابَ على مصراعيه للتدقيق في المنشورات

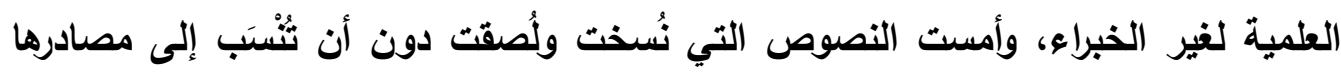

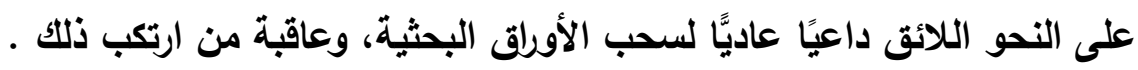

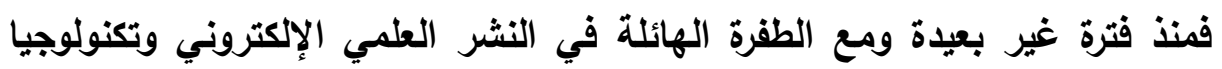
المعلومات وتوفر قواعد البيانات العلمية خاصة للإنتاج العلمي المنشور باللغة الانجليزية، ظهرت برامج الكشف عن الاقتباس وأصالة الإنتاج العلمي والتي أطلق عليها باللغة الإنجليزية Plagiarism software بينما في معظم البلدان العربية أطلق عليها برامج كثف السرقات الأدبية (الانتحال أو الاستلال) !!!. في الوقت الحالي هناك العديد من البرامج الالكترونية (بعضها مجاني والآخر غير مجاني) للكثف عن أصالة المؤلفات ونسب الاقتباس في الإنتاج الفكري والعلمي المنشور باللغة الانجليزية على مستوى العالم، فقد اتجهت مؤخرا العديد من الجامعات العالمية والمؤسسات البحثية إلى توفير بعض البرامج الفعالة وغير المجانية مثل علئل ithenticate and turnitin 
الاقتباس في الإنتاج العلمي، حيث تسمح هذه الخدمة - كما يرى مصمموها - بكشف الانتحال والتزوير العلمي والسرقات العلمية للأبحاث والمقالات العلمية والأطروحات الجامعية

‘ كثيرة هي برامج كشف الانتحال العلمي، ومنها المجاني ومنها المدفوع. وأهم هذه البرامج على الإطلاق :

Identicate \& Turnitin

والدكورة Identicate -1 هذا البرنامج المخصص لدور النشر العلمية وللباحثين والأساتذة ولطلبة الماجستير

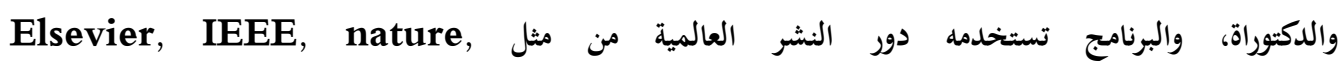
Springer,Wiley Blackwell

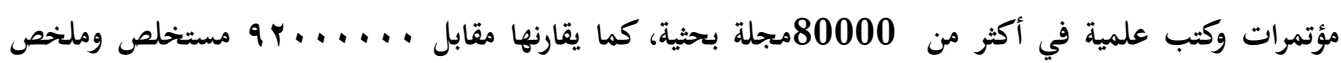

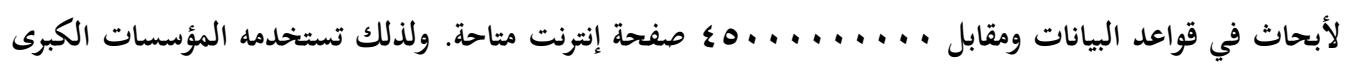
والجامعات الكبرى من مثل,Salford, Cambridge Harvard وغيرها، وكذلك المؤسسات القانونية ولئل

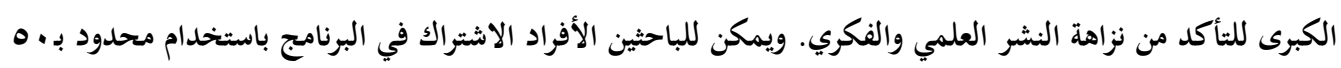

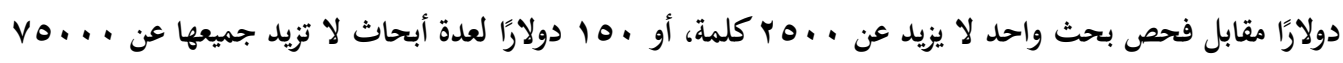

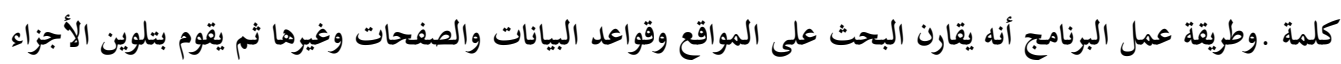

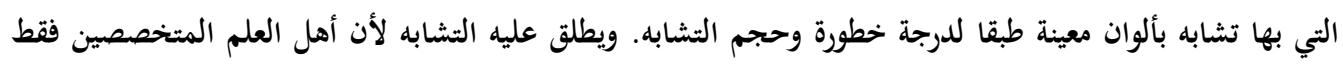

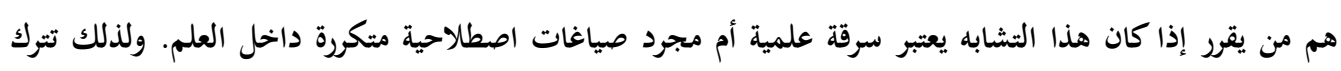

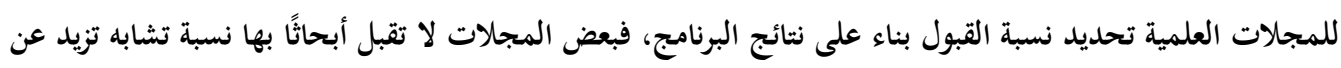

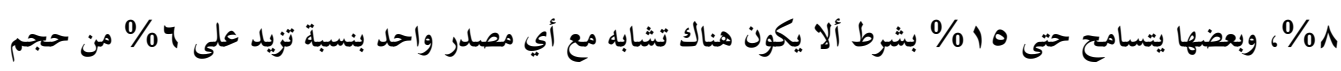

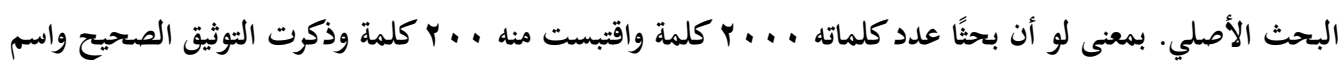

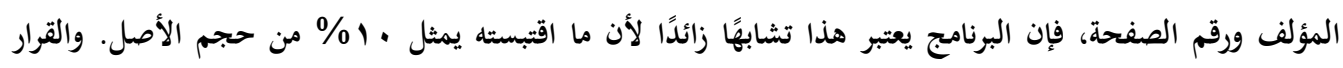

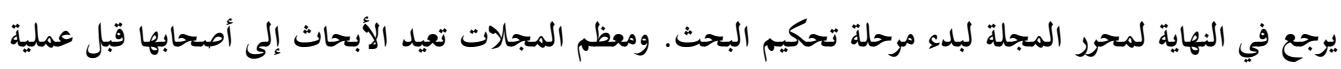

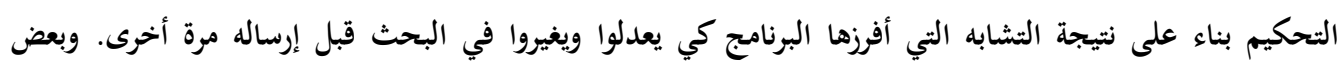

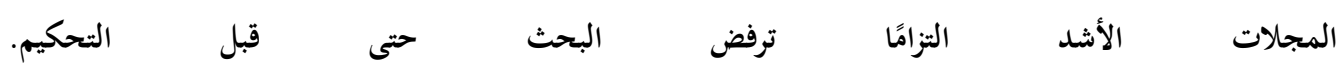
ومن نافلة القول أن برنامج ithenticate يقوم بهذه المقارنات في جميع اللغات لأنه يتعامل مع أكواد اللغات

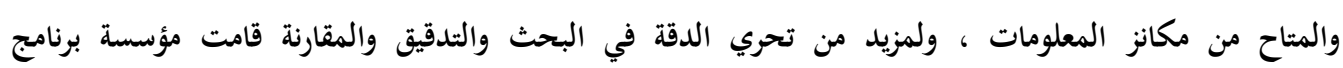
Cross Check بألاتحاد مع مؤسسة Crossref ithenticate powered by Ithenticate هو برمجية إلكترونية تعمل على شبكة الانترنت لكشف ومنع انتحال وسرقة المحتوى الإلكتروني : Turnitin - 2

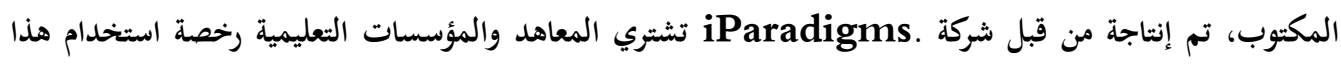

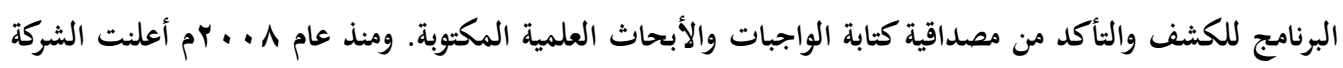

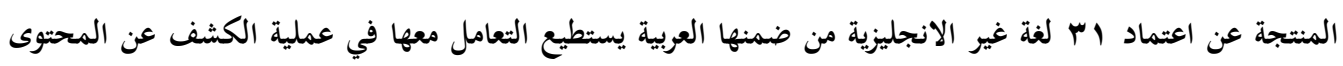


الصادرة باللغة العربية المتوفرة في قواعد البيانات الخاصة بها ”. من خلال تقريرا شاملا يوضح التشابه والتطابق في نصوص الأبحاث العلمية العربية والأطروحات الجامعية.

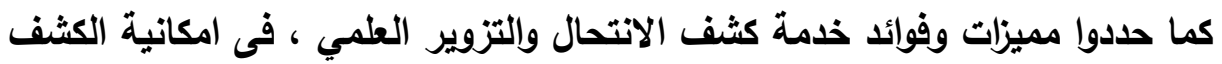
والحصول على تقرير يوضح نسبة التطابق التام أو الجزئي للانتاج العلمي المقام للجهة الناشرة، حماية حقوق الملكية للمؤلفين وللناشرين لاى قاعدة “معرفة”، وذلك عند وجود أي لوني اعتداء على حقوقهم الفكرية، مساعة الهيئات العلمية الناشرة في العالم العربي في التاكد والتحقق من الابحاث العلمية المقدمة لها ، اضافة الى تعزيز مبداً المسائة الاخلاقية وميثاق في فئي الثرف في الانتاج العلمي العربي ويالتالي حماية سمعة الجهات الناشرة والتاكد من نزاهة

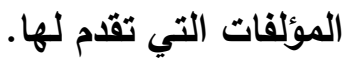

المنسوخ، تحتوي قاعدة ذلك البرنامج على اكثر من • ץ مليار صفحة انترنت، ما بين صفحات حالية ومؤرشفة

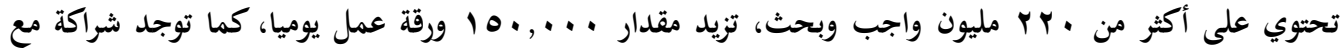

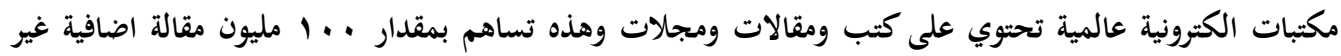

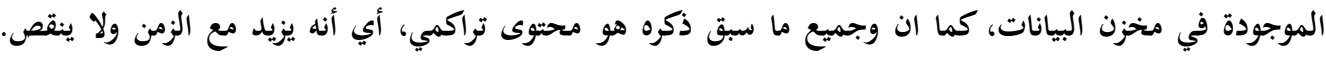

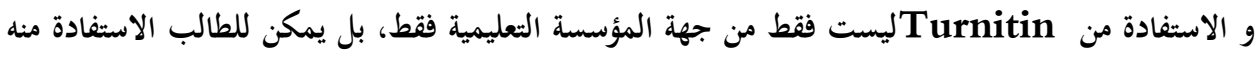

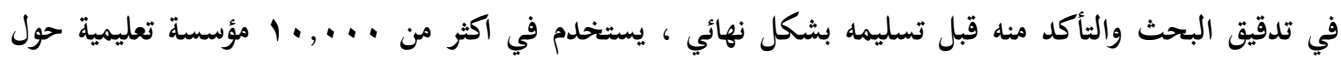

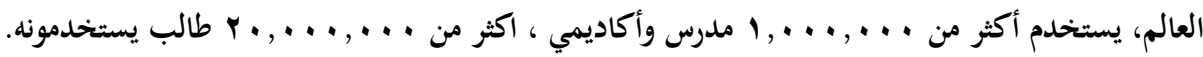

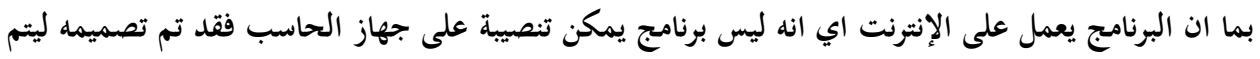

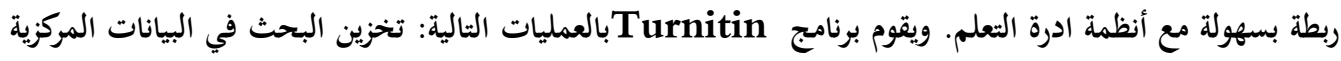

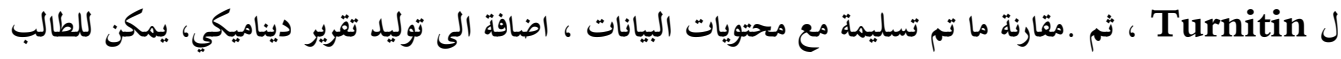

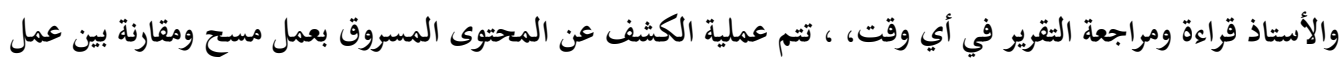

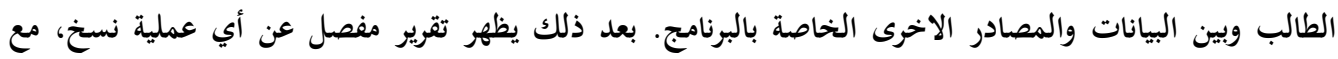

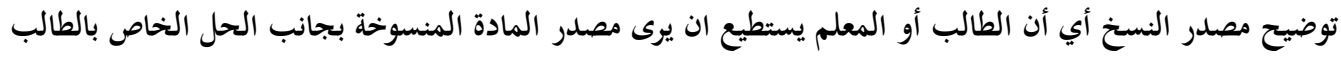

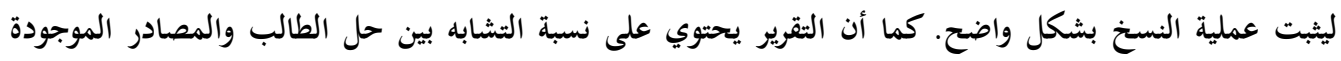

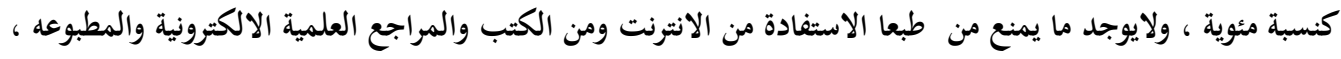

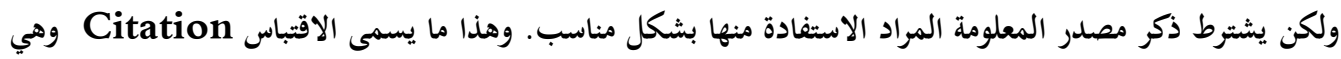

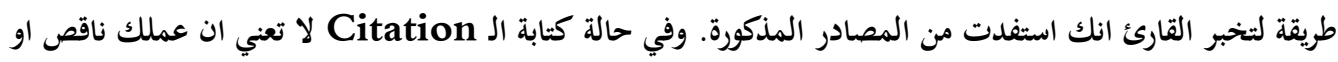

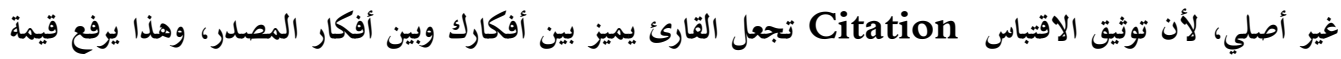


وتتمثل ابرز الخصائص الفنية لتلك الخدمة فى تحميل الوثائق واختبارها خلال دقائق ، وحفظ الثكل الاصلي للبحث الذي يتم تحميله، وتقديم تقرير دقيق عن نتائجه

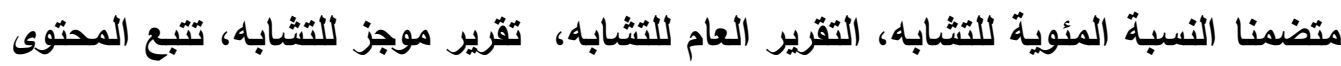
، اضافة الى خاصية تحميل وطباعة تقرير التشابه. هذه الخدمة يمكن ان تستقيد منها جهات عديدة من ابرزها ، الجامعات وهيئات

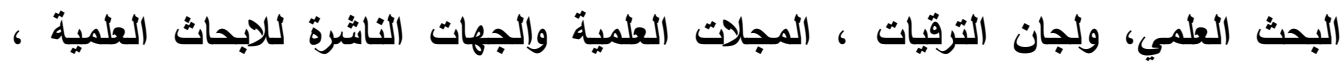

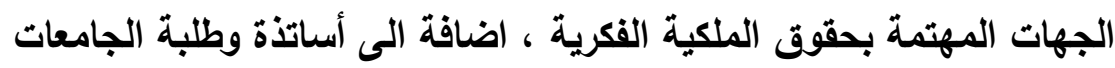
ولعل النظرة التحليلية الفاحصة لتلك البرامج تشير الى عدة ملاحظات عليها لابد من فن النظر فيها واخذها فى الاعتبار عن تطبيق تلكك البرامج حتى تؤدى تلك البرامج الغرض الذى وضعت من اجله بدقة وكفاعة تلك الملاحظات تتمثل فيما يلى : الحنى 1- الخلط فى تلك البرامج بين مفهومى الانتحال او السرقة العلمية ونسبة الاقتباس

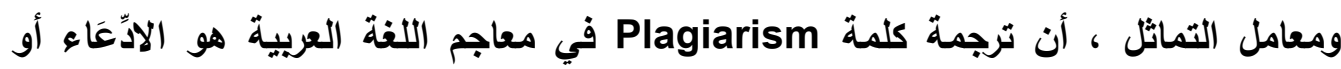
الانتحال أو القرصنة الأدبية، إلا ان كلمة Plagiarism في معاجم اللغة الإنجليزية (وهي اللغة الأصلية للكلمة) لها معاني أخرى مثل الاقتراض borrowing على سبيل المثال. ففي معظم البلدان العربية تعرف هذه البرامج الاكترونية Plagiarism software ببرامج الكثف عن السرقات الأدبية وهو تعريف أخرج هذه البرامج عن السياق الرئيسي والذي

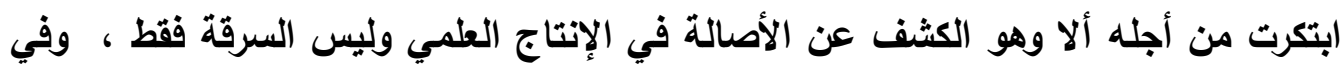
هذه الحالة أيضا فإن المؤلف الأي يستطيع أن يصل إلى القيمة صفر في نسبة الاقتباس

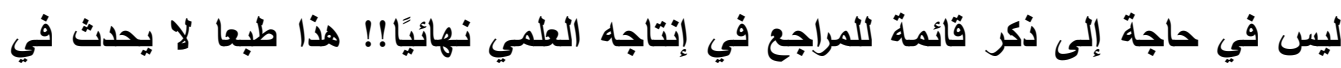

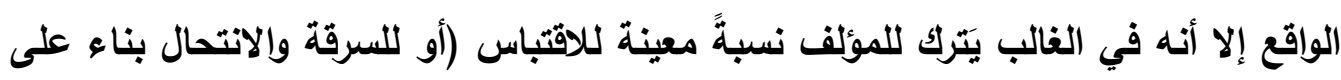
مسمى البرنامج) تختلف هذه النسبة من جامعة إلى أخرى ومن مؤسسة بحثية إلى أخرى!!!.

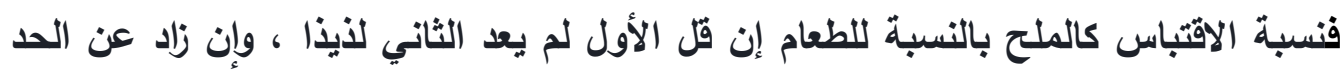
صار ممجوجا ، فعدد المراجع ونسبة الاقتباسات يجب ألا تنقص في البحث وألا تستخدم بإفراط. فتلتها تسيء إلى رصانته والإفراط في الاقتباس تضيع شخصية الباحث ويصمته.

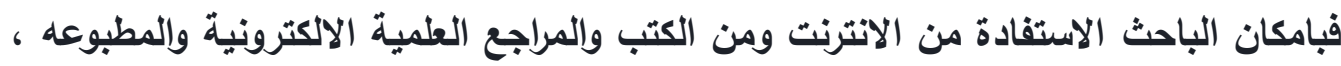
ولكن يشترط ذكر مصدر المعلومة المراد الاستفادة منها بشكل مناسب. وهذا ما يسمى 
الاقتباس Citation وهي طريقة لتخبر القارئ انك استفدت من المصادر المذكورة ، وفي حالة كتابة الـ Citation لا تعني ان عملك ناقص او غير أصلي، لأن توثيق الاقتباس Citation واذا كل طعام وكل متناول له فى حاجة الى نسبة مناسبة من الملح تختلف باختلاف

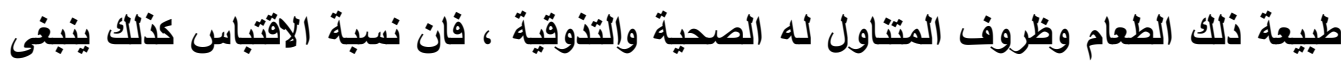
ان تختلف من تخصص الى تخصص اخر ، فالنسبة المسموح بها فى التخصصات العلمية لابد وان تختلف عن الدراسات المتعلقة بتحقيق كتب والتراث والتراجم وتخصصات علوم

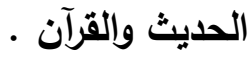

r - - إن الجامعات والمؤسسات البحثية واللجان العلمية للترقية تترك للباحث عند نشر الأورلق البحثية نسبة معينة (في غالب الأحيان من ·r\% إلى هr\%) للاقتباس (للسرقة الأدبية أو الانتحال بناء على مسمى البرنامج) - كما تترك المؤسسات الداعمة للبحث العلمي

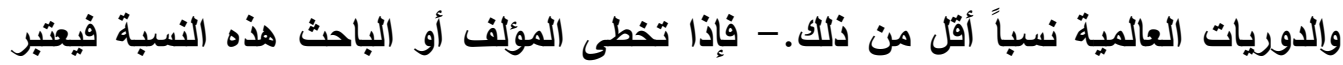
المؤلف أو الباحث (بناء على مسمى البرنامج) سارقاً ومنتحلاً وإذا لم يتخطى المؤلف هذه هنه النسبة فكاتت نسبة الاتبباس لاية أقل من النسبة المشترط ألا يتخطاها فهو في هذه الحالة برئ من تهمة السرقة!!!. ، وهذا امر فى حاجة الى اعادة نظر.

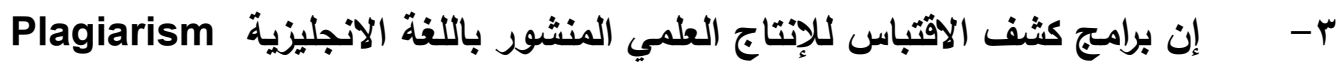
software المجانية المتاحة لكثف الاقتباس في الإنتاج العلمي نتائجها دون المستوى باعتراف معظم الباحثين على مستوى العالم. فليس من الغريب أنه عند الكثف عن الاقتباس بأحد البرامج تكون نسبة الاقتباس مختلفة تمام عن نسبة الاقتباس التي تظهر في برنامج آخر وذلك لنفس الورقة البحثية أو المنتج المعرفي!!. ع - اختلاف الهـف من تلك البرامج الخاصة بفصص وكثف نسب الاقتباس للانتاج العلمى المنشور باللغة الانجليزية عن ذلك الهدف للانتاج العلمى المنشور باللغة العربية فاذا كان الهدف هو كشف الانتحال والسرقات العلمية فإنه من المنطقي اعتبار أي نسبة تتعدى الصفر يجب أن تعتبر سرقةً علمية أو انتحالاً!! وفي هذه الحالة فأكاد أجزم بأن الإنتاج المعرفي العالمي بصفة عامة سوف يعتبر مسروقاً أو منتحلاً. ، اما اذا كان الهدف الكشف التف 
عن الأصالة وتقليل نسبة الاقتباس غير المرغوب ، فلا داعي مطلقا لتعريف هذه البرامج

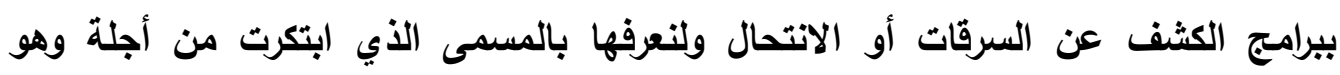
الكثف عن الأصالة في الإنتاج العلمي والعمل على رفع جودة الكتابة العلمية.

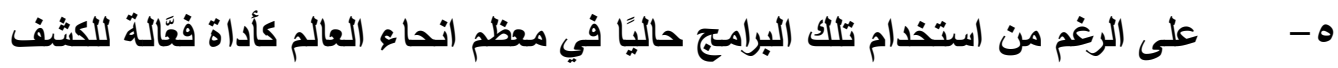
عن الاقتباس ، إلا ان المتمرسين على استخام تلك البرامج يعلمون جيدا أنه يمكن تغيير

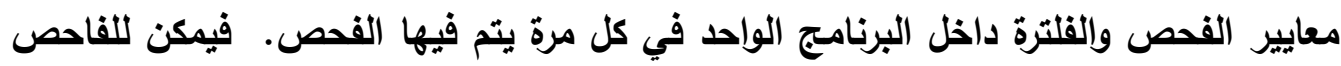

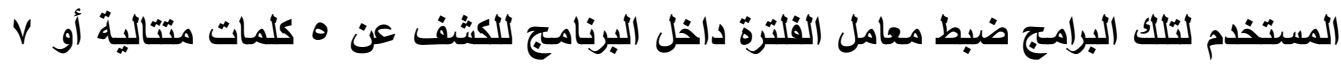

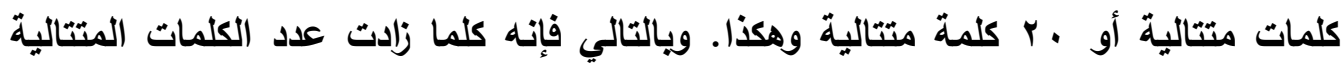
المستخدمة في عملية الفلترة كلما قلت نسبة الاقتباس التي يكتشفها البرنامج والعكس

صحيح.

إن علينا أن نتعلم من الجامعات البريطانية، حينما قامت بتجرية جديرة بالاقتداء،

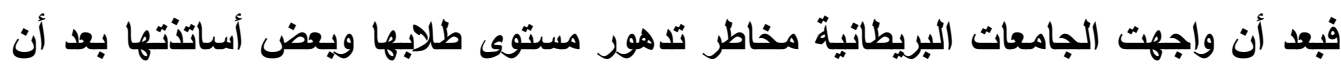
اشتهر كثيراً منهم بسرقة أبحاث وأفكار آخرين من خلال شبكة الإنترنت، في الوقت الذاني يتعين فيه على هؤلاء إطلاق العنان لأفكارهم ومخيلاتهم من أجل إثراء عملية التعات التعلم والارتقاء

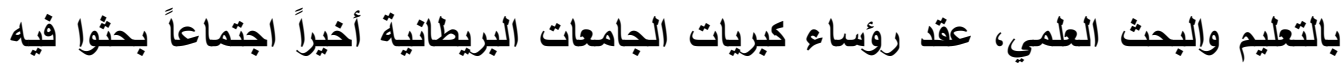

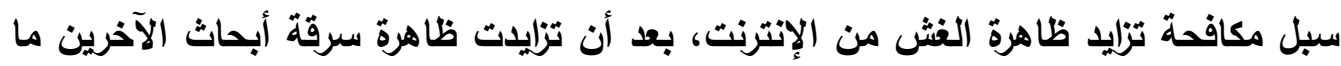

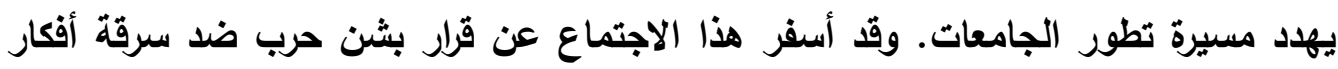
الآخرين، أطلق عليها (الحرب على جيل جوجل) نسبة إلى أكبر محرث بحث على على الإنترنت،

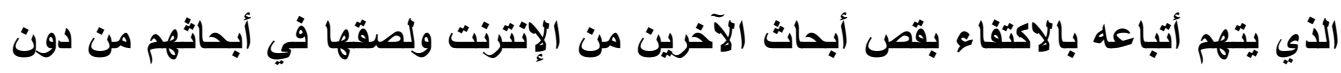
أدنى مجهود من جانب الطالب. لذا اتفقت الجامعات البريطانية الكبرى مثل أكسفورد

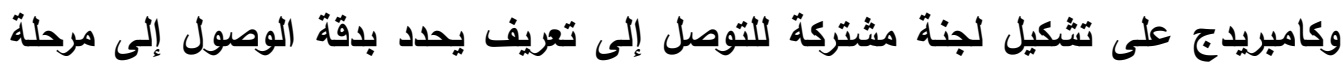
توجيه الاتهام بالغش وسرقة الملكية الفكرية، فبعض الجامعات البريطانية طالبت بتوجيه

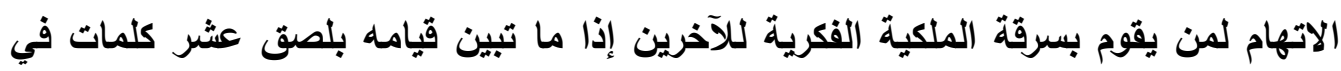
بحثه تتثابه بصورة طبق الأصل من عثر كلمات موجودة في بحث منثور على شبكة الإنترنت، في حين طالبت جامعات أخرى بتوجيه الاتهام بالغش في حال تبين أنه اقتبس ثُلاث جمل من بحث آخر من دون أن يذكر بوضوح المصدر المنقول عنه. 
צ- ان استخام تلك البرامج للكثف عن نسب الاقتباس بالنسبة للإنتاج المعرفي المنثور باللغة العربية، عملية غير قابلة للتنفيذ واقعيا فى الوقت الحالى ، نظرا لعدم وجود قواعد بيانات تشمل الإنتاج العلمي العربي ، وحتى تتوفز قواعد بيانات عربية شاملة للإنتاج

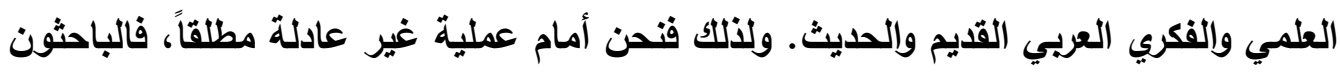

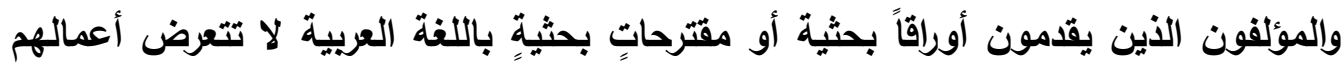
إلى عملية كثف الاقتباس بنفس المستوى الذي تتعرض لله الأعمال المقدمة باللغة

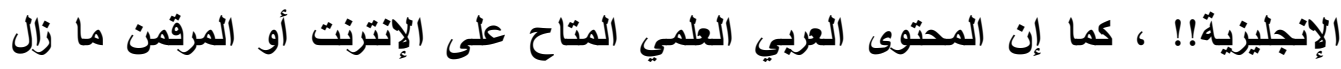

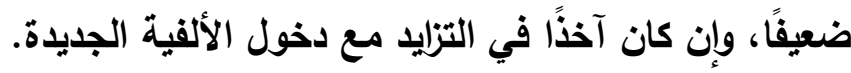

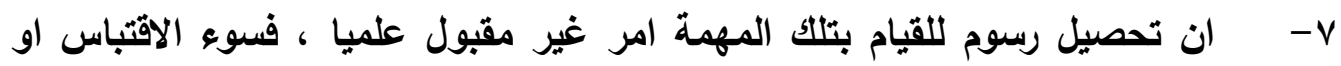

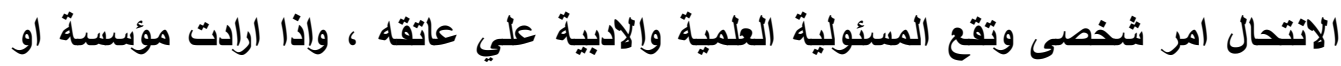
جهة علمية القيام بذلك فالافضل الاتكون بدون رسوم حتى لاتتحول الى عملية تجارية

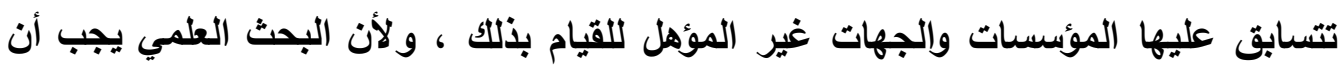
يرتكز في المقام الأول على الثقة المتبادلة، وفيما عدا ذلك فئل فإن "البرمجيات مفيدة كجهاز

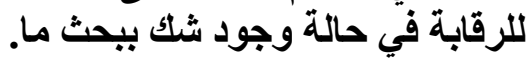

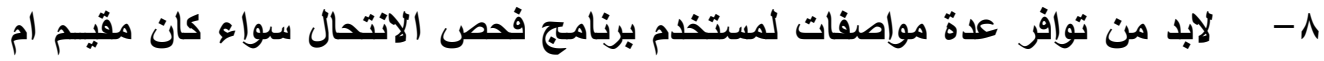

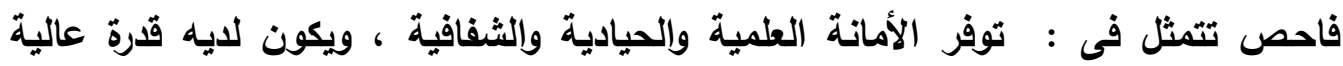
باستخدام الحاسوب والمعرفة الكامله باستخدام البرنامج والالمام بكل إمكانياته في الفحص، وكذلك درايته بخيارات الفحص حيث يجب ان تحدد بغناية وتوحيدها وامكانيات البحث، الاستثاءات في المقارنات وهل يتم حفظ الأورلق البحثية في قاعدة البيانات، كما يجب البان الافضل - ان يكون مستخدم البرنامج (الفاحص) هو ذا اختصاص في (البحث، الرسالة أو الإطروحة) المراد فحصها ليتم (ستبعاد التثابة من النسبة. 9- - ان تلك البرمجيات قد تفثل فى كثف الانتحال اذا تم تعديل النص او اعادة الصياغة لانها تعتمد على مقارنة النصوص بما هو موجود فى قاعدة بيانات البرنامج

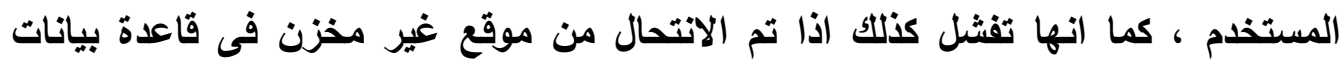

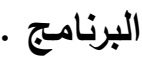

• - ان الاعتماد على تلك البرمجيات لكثف الانتحال للبحوث المقدمة باللغة العربية مهمة صعبة وليست سهلة ، نظرا لان تلك البرمجيات المتاحة صممت لكثف الانتحالات 
والاقتباسات للبحوث المنشورة باللغة الانجليزية ، ولاتاخذ فى اعتبارها طبيعة وخصائص اللغات الاخرى والتى منها اللغة العربية تلك اللغة التى تتميز بصعوية تراكيبها وكلماتها اللغوية ، ان لغة البرمجة الاساسية لتلك البرمجيات هى اللغة الانجليزية وسيكون من الصعوية استخدام برنامج صمم باللغة الانجليزية ثم يتم تعريبه لاستخدامه في اللغة العربية ،

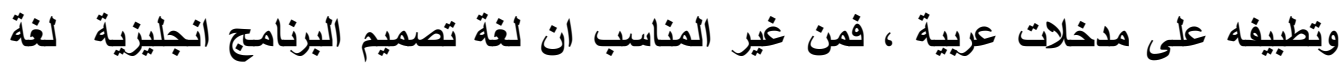
استخدامه عربية ، الامر الذى يتطلب ضرورة تقييم كفاءة تلك البرامج فى كثف الانتحال

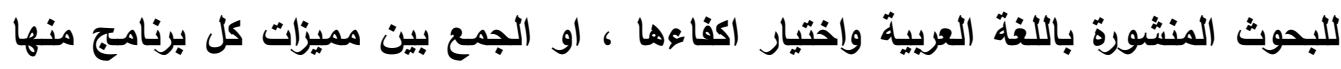

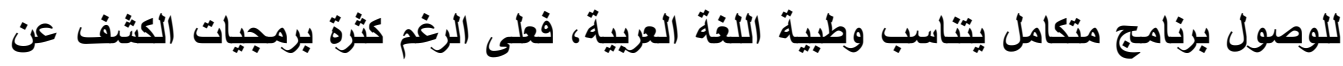
الانتحال وتنوعها ، فانتا لازلنا فى حاجة الى برمجيات قادرة على فحص البحوث المكتوية باللغة العربية غير المستخدمة باللغات الاخرى.

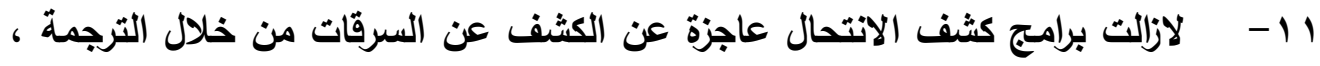
فكثف سرقة الترجمات يعد واحدة من أصعب المشاكل للتظلب عليها واكثافها ، حيث أصبحت

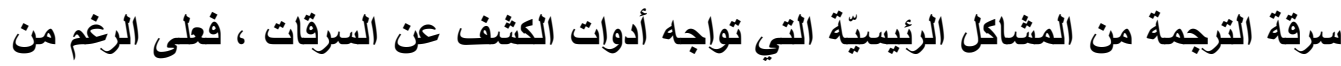
انتشار العديد من برامج كثف الانتحال وتتوعها ، فما تزال سرقة الترجمة عملية غير التئه

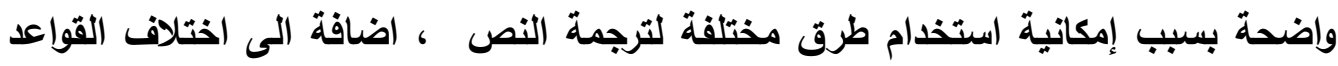

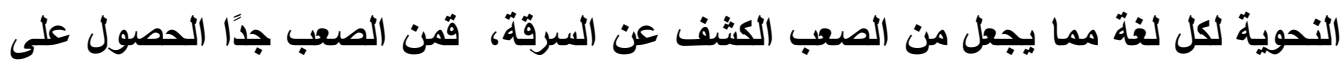
ترجمة جيدة بسبب صعوية إيجاد أدوات ترجمة عالية الجودة ، كما أنها تتطلب الكثير من الكن

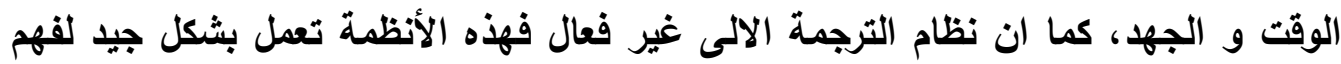

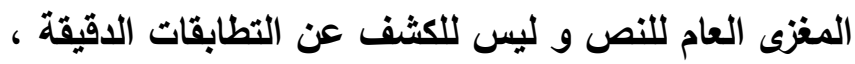
ولذلك هنالك العديد من الناشرين المخادعين و الكُّاب غير الأخلاقِيين، لايهر

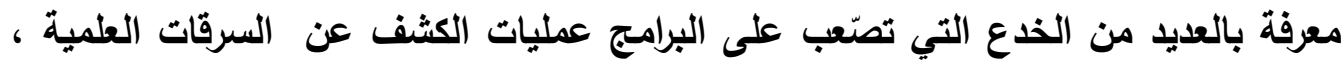
على سبيل المثال : ملفات PDF عادة تكون مصنوعة من عدة طبقات. توجد الطبقة المرئية و طبقة النص، من الممكن تغيير طبقة النص غير المرئي عن طريق تغيير جميع الأحرف

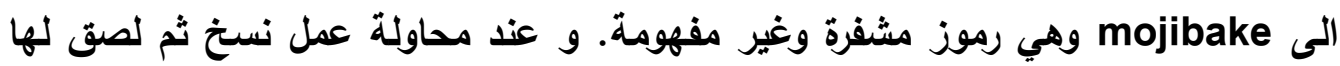
على الدفكرة ستحصل على أحرف مبهمة. ولهذا السبب، لن تستطيع أدوات الكثف الآلية 
قراءة النص. بالإضافة إلى أن استعمال هذه الطريقة سيصعب عملية إيجاد منشورات الكاتب بواسطة محرك البحث قوقل.

ولذك ففي الكثير من الحالات، وغالبا ما يتم الكثف عن تلك السرقات بواسطة المعلم

أو المدرس، وذلك لأنه يلاحظ التغير في أسلوب كتابة الطالب. وفي الحقيقة، يعد الإنسان من

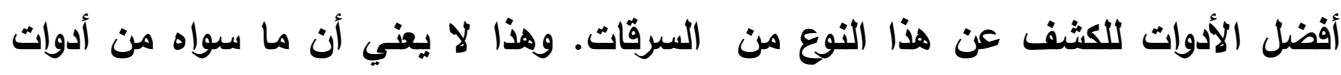

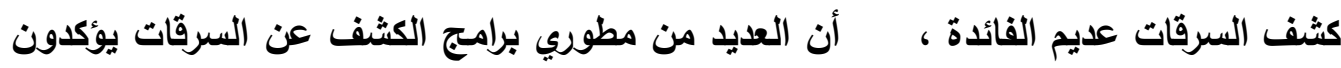
على أن العرقة أصبحت واسعة في معظم المقالات الإنجليزية لارجة أصبح العملاء يطالبونهم للكثف عنها بأي طريقة ممكنة.

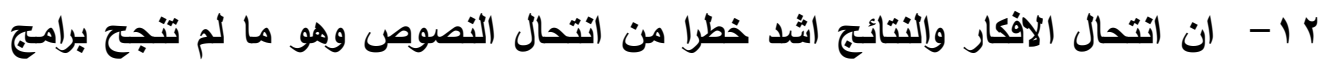
كثف الانتحال المتاحة فى الكثف عنه بدقة. rا

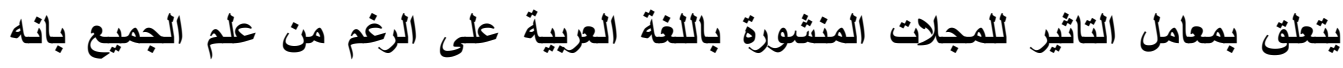

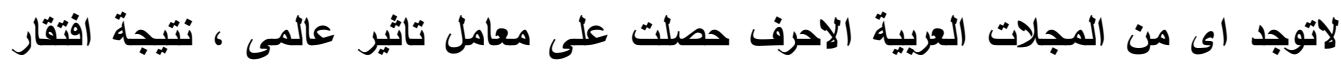

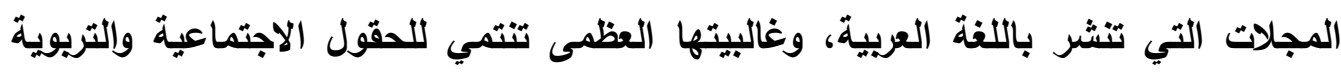
والانسانيات، لجهة تصنيفية موحدة لتحديد معاملات تأثير لها، والذى يعد من أهم مشاكل

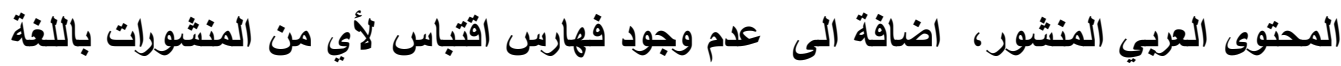

العربية

ولعل فى تفعيل ودعم مشروع معامل التاثير العربى باب امل للتظلب على تلكي

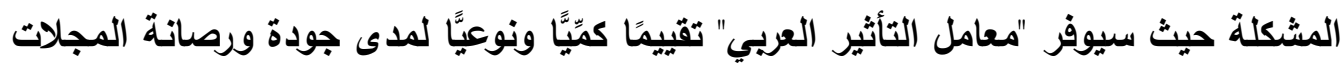

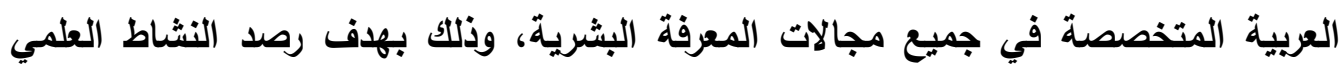

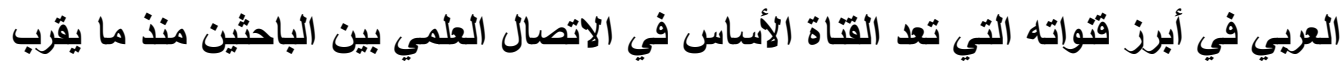

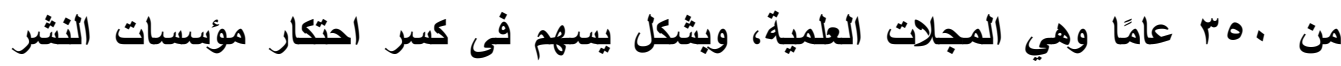

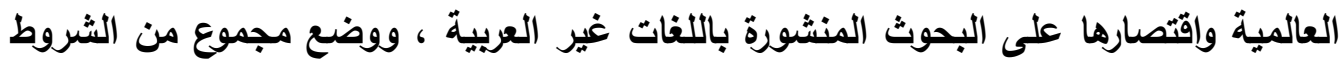
المجحفة لأوعية النشر باللغة العربية ، والتي قد لا يكون لها علاقةة بجودة النشر العلمي، اضافة الى اهميته في اعادة شيء من الاعتبار إلى لغتنا العربية ، ويضع المتميز من لإنها

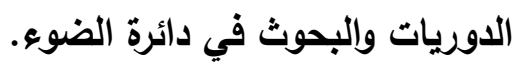


برامج كشف الانتحال للبحوث المنشورة باللغة العربية بين الحقيقة والوهم.
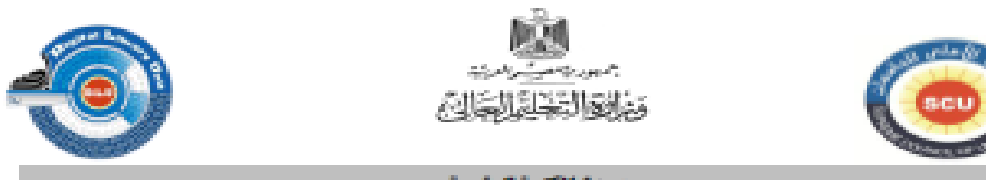

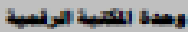

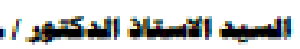

تمبة طبوبة و وعد ........

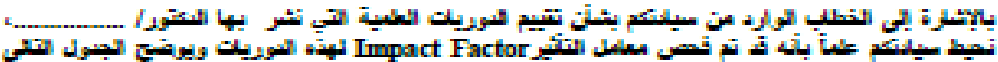

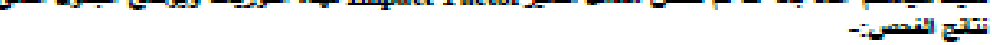

\begin{tabular}{|c|c|c|c|c|c|c|c|c|}
\hline & Journal Titte & ISSN & Publisher & $\begin{array}{l}\text { Impact } \\
\text { Factor }\end{array}$ & Quartile & SUR & Quartile & Clessification \\
\hline 1 & & & & & & & & \\
\hline 2 & & & & & & & & \\
\hline$r$ & & & & & & & & \\
\hline f & & & & & & & & \\
\hline 5 & & & & & & & & \\
\hline 6 & & & & & & & & \\
\hline
\end{tabular}

\section{مديز وحدة الإكتيات اللزمبية}

أ. 


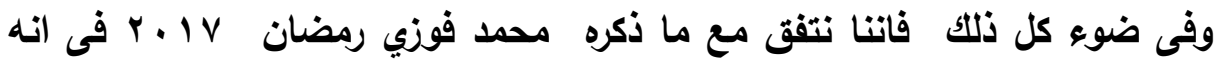
لكى نتخلص من العشوائية الحالية من حيث المعنى والمضمون في الكشف عن الاقتباس في النشر العلمي ، وتحقق تلك العملية الاهداف المرجو منها ، وتتحول تلك العملية من وهم الى حقيقة علينا مراعاة الاتى: ا- توفير قواعد بيانات شاملة لكل الانتاج العلمى العربى ، والا تقتصر على الانتاج

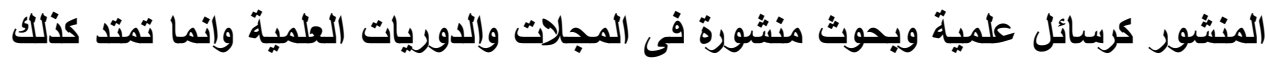
الى الكتب والمقالات والاعمال الفنية العلمية المنشورة بل تمتد لتشمل كل انتاج فكرى ومعرفى ، فللاسف مازال قطاعا كبيرا من مجتمعنا العلمي يعيش عصور ما قبل

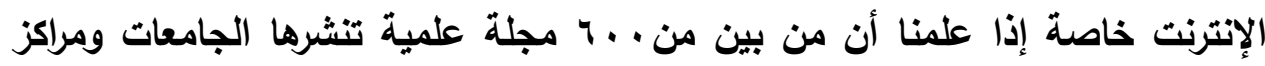
البحوث المصرية ، ـ م مجلة فقط مدرجة ضمن النشر الدولي ومتوافرة علي الثبكة العنكبوتية كي يطلع عليها علماء وياحثو العالم ، تلك النظرة المحدودة وعدم الاكتراث بقيمة النشر العلمي أسهمت بدورها في انتشار الكثير من المفاهيم الخاطئة والمرفوضة دوليا مثل نقل البحوث دون الإثارة لمصادرها وادعاء حداثة الأفكار المنشورة من قبل كاتبيها r- توحيد البرنامج المستخدم في الكثف عن الاستلال (نظرا لكثرة البرامج واختلاف نتائجها). ب- توحيد أجزاء الورقة البحثية أو المقترح البحثي التي سيتم الكشف عن الاستلال بها (فمن المفترض ان لا يتم الكشف عن الاستلال في قائمة المراجع على سبيل المثال). ع - توحيد خيارات ومعاملات الفحص والقلترة المستخدمة في الكثف عن الاقتباس. ه- توحيد نسب الاقتباس المسموح بها في جميع الدوريات العلمية والمؤسسات البحثية

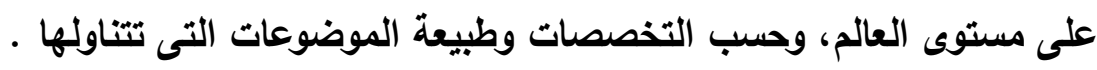
צ- اتاحة البرنامج المستخدم في الكشف عن الاقتباس للباحثين والمؤلفين بصورة مجانية ما 


\section{المراجع}

1- اشرف امين : الأهرام يكتشف عبر محرك البحث لدوريات:ELSEVIER 25 باحثا بالجامعات

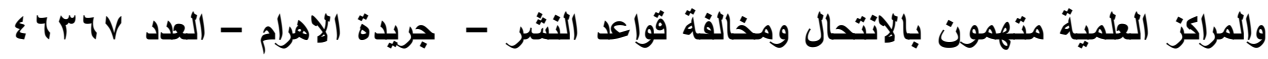

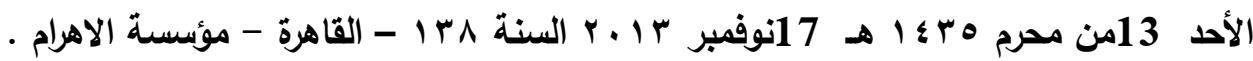
r- الأقتباس العلمي و برامج قياس نسبة الاقتباس وكثف الانتحال والسرقات العلمية متاح على https://ab7aaaas.blogspot.com/2017/08/blog-post_16.html r- برافين تثشادا : لا تستدعي كافة الانتحالات العلمية سَحْبَ الأبحاث المنشورة متاح على https://arabicedition.nature.com/journal/2014/08/511127a Identicate \& Turnitin برامج قياس نسبة الاقتباس وكثف الانتحال والعرقات العلمية https://www.facebook.com/higher.studies.mag/posts/1423231367790573 متاح على

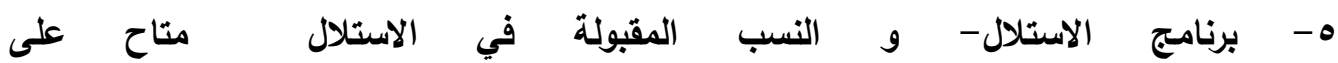
http://www.uoanbar.edu.iq/HumanitarianEducationCollege/News_Details.php?ID=20 צ- جمال على الدهثان : تقرير عن ندوة الانتحال العلمي: (المفهوم، المظاهر، الأسباب، وأساليب الكثف عنه) والتى عقدت بكلية التربية جامعة المنوفية بالتعاون مغ المكتبة الرقمية بالجامعة

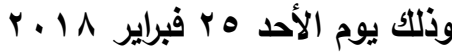
- - جمال على الدهشان : محارية السرقات العلمية مدخلا لتحقيق جودة البحث التربوى العربى فى عصر المعلوماتية - مؤتمر "التعليم فى الوطن العربى ، نحو نظام تعليمى متميز" المنعقد

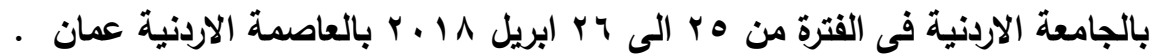
1- - جمال على الدهشان : نحو معامل تاثير عربى لجودة وتقييم المجلات والبحوث العلمية المنشورة باللغة العربية ، الضرورات ، والمتطلبات - المجلة الدولية للبحوث فى العلوم التربوية I International Journal of Research in Educational Sciences

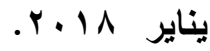
9 - حسن على دواح : اوجه الفساد فى مجال البحث العلمى : الانواع الاسباب الانعكاسات -

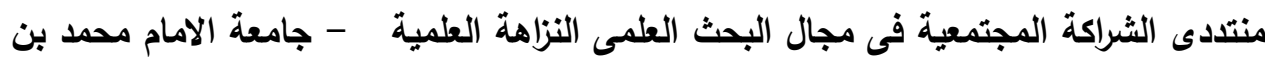

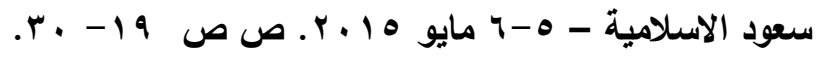

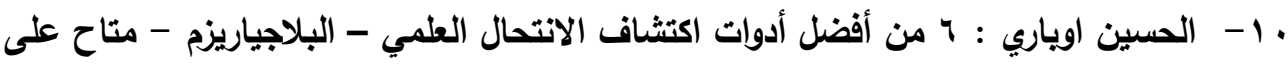




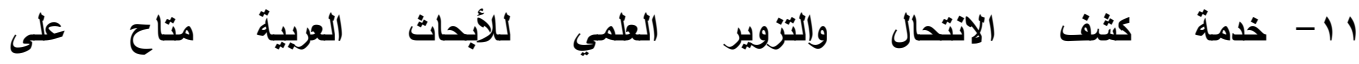
file:///E:/\%D8\%A8\%D8\%AD\%D9\%88\%D8\%AB/\%D8\%A7\%D9\%84\%D 9\%86\%D8\%B2\%D8\%A7\%D9\%87\%D8\%A9\%20\%D8\%

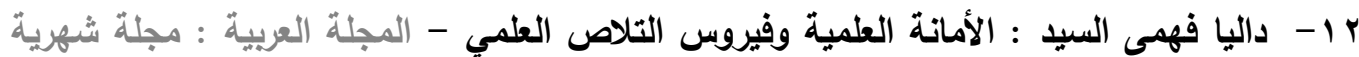

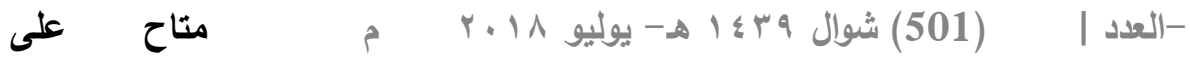
http://www.arabicmagazine.com/arabic/ArticleDetails.aspx?id=1642\# .TzbzIGoc9zU.facebook r - سرقة الترجمة تحد جديد امام برامج كشف السرقة الادبية متاح على http://ziid.net/tech/translation-plagiarism-detection ء ا- المجلس الاعلى للجامعات ، وحدة المكتبات الرقمية : دليل فحص النزاهة الاكاديمية - وزارة

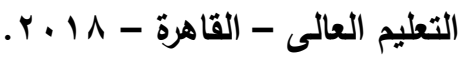
10- محمد فوزي رمضان حسانين : إعادة النَّر .. بين حُقوق النّشر والحَقِّ في المعلومة متاح

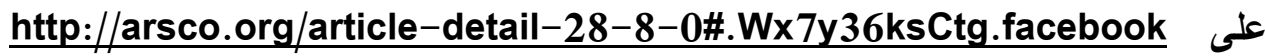

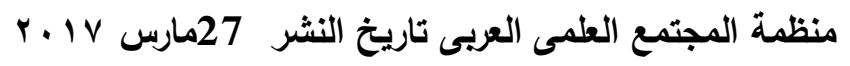
17 - محمد فوزي رمضان حسانين : الاقتباس في النشر العلمي: بين الأصالة والسرقة - متاح على تانئ

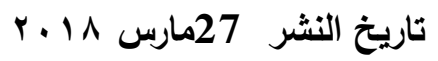
IV - نوف محمد الظاهرى واخرون : تقييم اداء البرامج العالمية لكثف السرقات العلمية باللغة العربية للابحاث الجامعية - منتدى الثراكة المجتمعية فى مجال البحث العلمى - منتددى

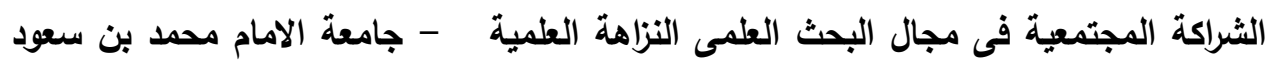

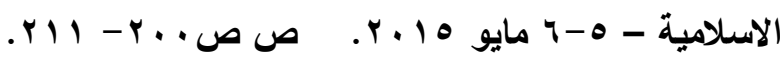

18- Stephan dahl: : Turnitin, The student perspective on using Plagiarism detection software, Active Learning in Higher Education, vol 8 (2) ,2015,173- 191.

19-Russell K. Baker et ail : An Evaluation of The Effectiveness of Turnitin. Com As A Tool For Reducing Plagiarism In Graduate Student Term Papers,college Teaching Methods\& Styles Journal,vol.4(9), September 2008> 